\title{
ESTUDO DA DISCIPLINA DE FARMACOLOGIA NOS CURSOS DE GRADUAÇÃO EM FARMÁCIA DA REGIÃO SUDESTE DO BRASIL
}

Ronilson Ferreira FREITAS ${ }^{1}$

Edileuza da Conceição SANTOS ${ }^{2}$

Márcia Rita Guedes RIBEIRO

Mara Regina Campos MORAES ${ }^{2}$

Vinícius Lopes CANTUÁRIA ${ }^{2}$

Bernat Vinolas PRAT ${ }^{3}$

${ }^{1}$ Professor Substituto do Departamento de Farmácia e Mestrando em Saúde, Sociedade e Ambiente da Universidade Federal dos Vales do Jequitinhonha e Mucuri - UFVJM.

${ }^{2}$ Acadêmicos do Curso de Graduação em Farmácia da Universidade Federal dos Vales do Jequitinhonha e Mucuri UFVJM.

${ }^{3}$ Professor do Mestrado em Saúde, Sociedade e Ambiente da Universidade Federal dos Vales do Jequitinhonha e Mucuri - UFVJM através do Programa Jovem Talento Nível A (Programa Ciência sem Fronteiras).

Recebido em: 23/01/2016 - Aprovado em: 23/06/2016 - Disponibilizado em: 30/07/2016

\begin{abstract}
RESUMO
Dentre as atividades da profissão farmacêutica, destaca-se a pesquisa, o desenvolvimento e a produção de novas drogas, utilizando-se como fonte plantas, animais e minerais, o que torna a Farmacologia uma das principais disciplinas do Curso de Farmácia, uma vez que estuda as substâncias que interagem com sistemas vivos por meio de processos químicos, ligando-se especificamente a moléculas reguladoras e ativando ou inibindo processos corporais normais. Diante desse pressuposto, este trabalho objetivou avaliar o estudo da disciplina de Farmacologia nos cursos de graduação em Farmácia da região sudeste do Brasil. Pela busca interativa, selecionaram-se os cursos de Farmácia cadastrados nos estados do sudeste do Brasil: Minas Gerais, Espirito Santo, Rio de Janeiro e São Paulo. Foram avaliados pontos como a nomenclatura que a disciplina recebe, carga horária total da disciplina, e a sua distribuição em aulas teóricas e/ou práticas, além do período em que a disciplina é oferecida. A análise das informações obtidas com a pesquisa demonstrou a necessidade de uma reavaliação da grade curricular para a oferta de disciplinas relacionadas com a farmacologia e, consequente dos seus planos de ensino e ementa da disciplina, nos cursos de graduação em Farmácia do Sudeste do Brasil.

Palavras-chave: Graduação em Farmácia; Farmacologia; Região Sudeste do Brasil.

\section{STUDY OF PHARMACOLOGY COURSES FOR UNDERGRADUATE PHARMACY PROGRAMSIN SOUTHEAST BRAZIL}

\begin{abstract}
Among the activities of a pharmaceutical professional, the research, development, and production of new drugs that use plants, animals, and minerals as raw material are highlighted. This makes pharmacology one of the priorities for Pharmacy Programs, since it studies the substances that interact with living systems through chemical processes, specifically by binding regulatory molecules and activating or inhibiting normal body processes. Given this assumption, this study aimed to evaluate the study of Pharmacology Courses for Undergraduate Pharmacy Programs in Southeast Brazil. Through interactive search, we selected all registered Undergraduate Pharmacy Programs in all Southeast Brazilian states: Minas Gerais, Espirito Santo, Rio de Janeiro and São Paulo. There were evaluated points as the course nomenclature, number of credit hours, its distribution of lectures and laboratory classes, and the period in which the course is offered. The analysis of the information obtained from the research showed the need for a reassessment of the curriculum for the supply of courses related to pharmacology and their syllabus, for Undergraduate Pharmacy Programs in Southeast Brazil.
\end{abstract}

Keywords: Undergraduate Pharmacy Program; Pharmacology; Southeast Brazil. 


\section{INTRODUÇÃO}

O Curso de Graduação em Farmácia é um dos mais antigos da história, e ao longo de sua trajetória, os profissionais farmacêuticos transcorreram crises, mudanças e evoluções, passando por momentos históricos importantes, determinados pelos diferentes cenários políticos, econômicos e sociais (RICIERI et al., 2006).

A Resolução CNE/CES n ${ }^{\circ}$ 2, de 19 de fevereiro de 2002, que institui as Diretrizes Curriculares Nacionais do Curso de Graduação em Farmácia, foi um marco na produção de mudanças no processo de formação dos profissionais farmacêuticos, sendo considerada como um caminho que flexibilizou as regras para a organização do curso, favorecendo a construção de maiores compromissos das Instituições de Ensino Superior com o profissional e a sociedade (BERMOND et al. 2008).

De acordo com essa resolução, o Curso de Graduação em Farmácia tem como objetivo, graduar o Farmacêutico com formação generalista, humanista, crítica e reflexiva, para atuar em todos os níveis de atenção à saúde, seja diretamente ou não com o paciente, com base no rigor científico e intelectual, pautado em princípios éticos e na compreensão da realidade social, cultural e econômica do seu meio, dirigindo sua atuação para a transformação da realidade em benefício da sociedade.
Dentre as áreas de atuação do farmacêutico generalista, tem o estudo da manipulação de fármacos, da criação e aplicação de métodos de controle de qualidade, da prevenção e promoção à saúde da população, principalmente quando diz respeito ao uso racional do medicamento, da criação e aplicação de métodos de identificação e dosagem de tóxicos, além de atividades como a pesquisa, o desenvolvimento e a produção de novas drogas, utilizando-se como fonte plantas, animais e minerais (PESSOA, 2010).

Neste sentindo, a Farmacologia se torna umas das principais disciplinas do Curso de Farmácia, uma vez que estuda as substâncias que interagem com sistemas vivos por meio de processos químicos, ligando-se especificamente a moléculas reguladoras e ativando ou inibindo processos corporais normais (STOLF; DREIFUSS; VIEIRA, 2011).

O novo currículo do curso de farmácia vem sendo questionado e analisado por profissionais e educadores, dentre os problemas que frequentemente tem sido discutido, diz respeito à carga horária do curso, em especial das disciplinas oferecidas, como por exemplo, a carga horária da disciplina de farmacologia, que deve ser condizente com o peso de sua importância na formação dos farmacêuticos. Tais problemas devem ser resolvidos com o intuito de buscar melhor uniformidade, lembrando, que se 
devem manter possibilidades de inovação dentro do curso, para que assim, possam buscar atender as peculiaridades regionais (CARDOSO et al., 2011). As Diretrizes Curriculares Nacionais, através das habilidades e competências descritas para o farmacêutico, impõem uma dinâmica de ensino que se adequa à aprendizagem significativa (FERNANDES et al., 2008).

Nessa conjuntura, faz-se importante a realização de estudos que avaliam a qualidade da disciplina de farmacologia que é oferecida pelos cursos de graduação em farmácia, e assim, esse estudo objetivou avaliar o estudo da disciplina de Farmacologia nos cursos de graduação em Farmácia da região sudeste do Brasil.

\section{METODOLOGIA}

Estudo descritivo sobre a oferta da disciplina de Farmacologia nos cursos de graduação em Farmácia na região sudeste do Brasil, em análises feitas no ano de 2015. Inicialmente, consultou-se o sistema "EMEC", Instituições de Ensino Superior e Cursos cadastrados, na "homepage" do Ministério da Educação e Cultura (www.emec.mec.gov.br).

Pela busca interativa, selecionou-se os cursos de Farmácia cadastrados nos estados do sudeste do Brasil: Espirito Santo, Minas Gerais, Rio de Janeiro e São Paulo. A partir da relação obtida, os detalhes das estruturas curriculares de cada curso foram levantados por meio do acesso direto ao website da Instituição, além do contato via e-mail para completar as informações. As informações analisadas referiram-se à:

- Nome da Instituição

- Nomenclatura da disciplina de Farmacologia

- Carga horária total da disciplina

- A sua distribuição em aulas teóricas e/ou práticas

- Período da sua inserção durante o curso

Os resultados foram organizados em gráficos e tabelas, e analisados de forma global, sendo apresentados através de valores absolutos e/ou porcentagens.

\section{RESULTADOS E DISCUSSÃO}

Atualmente, existem 171 Instituições de Ensino Superior que oferecemo curso de Farmácia e estão cadastradas no sistema "eMEC". Porém, foram avaliadas um total de 122 Instituições, o que corresponde a $71 \%$ dos cursos de farmácia oferecidos na região sudeste do Brasil (Gráfico 1), o que possibilitou observar que a disciplina de farmacologia possui diferentes nomenclaturas. Entretanto, no que se refere à correspondência entre o nome da disciplina e o conteúdo que é ministrado, não foi possível avaliar essa relação, uma vez que não foi analisada a ementa das disciplinas. 
Gráfico 1 - Número total de instituições cadastradas sistema "e-MEC" versus instituições avaliadas.

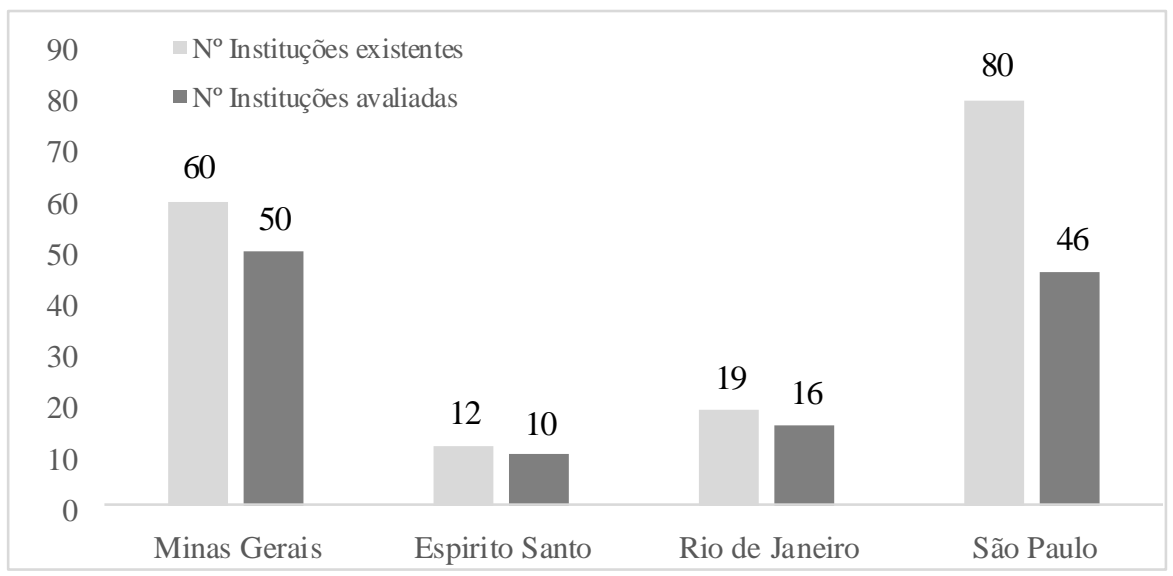

Embora a distribuição da carga horária possa indicar que a farmacologia, na maioria dos casos é capaz de contemplar os conteúdos necessários à disciplina, foi possível observar uma variação grande na carga horária total (média de 206,05 h e desvio padrão de 106,11 h) da disciplina entre uma instituição e outra, sendo que existem instituições que a carga horária total foi inferior a 100 horas, o que poderá comprometer o processo de ensinoaprendizagem do aluno (Gráfico 2).

A farmacologia é uma ciência que estuda as interações dos fármacos com os diversos sistemas biológicos do corpo humano. Diante do fato de que essas interações podem ser benéficas (efeitos terapêuticos), e também maléficas (efeitos adverso), faz-se necessário o conhecimento completo do conteúdo que a abrange pelo profissional que lida com as consequências do uso dessas substâncias, sendo assim, é importante que a carga horária da disciplina seja compatível com a importância e a quantidade de conteúdo que é ministrado pela disciplina (DIAS, 2016), sendo que os conhecimentos básicos neste campo são de suma importância para a compreensão das ações dos medicamentos (MOREIRA; GUIMARÃES, 2007).

Apesar da mudança do paradigma de que o farmacêutico é o "profissional do medicamento" para o de que o farmacêutico é o profissional voltado para o usuário do medicamento, de acordo com Adib (2015), ainda assim, sem o conhecimento detalhado da farmacologia é impossível que o profissional possa atuar na atenção ao usuário, uma vez que essa atenção é completamente baseada nos efeitos das drogas que estão sendo utilizadas por esse usuário juntamente com todos os fatores externos a ele que afetam o tratamento terapêutico.

Nesse contexto, sendo a farmacologia útil para a prática aquela que é estudada na 
sua base e levada até a sua aplicação à clínica, é importante ressaltar também que além da decadência na estruturação das disciplinas de farmacologia nos cursos de farmácia, problemas ainda maiores são o ensino dessa disciplina aplicada à área social por assim dizer, e a carga-horária dedicada a essas disciplinas na estruturação das grades dos cursos de farmácia.
Visto que a necessidade do ensino da disciplina de farmacologia envolve muito mais detalhes, a carga-horária a ela destinada deve ser criteriosamente analisada. De acordo com os dados obtidos na presente pesquisa é possível perceber a enorme diferença entre instituições de ensino no que diz respeito ao ensino de farmacologia, o que corrobora com uma formação imensamente discrepante entre os profissionais da região sudeste do Brasil.

Gráfico 2 - Carga horária da disciplina de Farmacologia dos Cursos de Farmácia da região sudeste do Brasil.

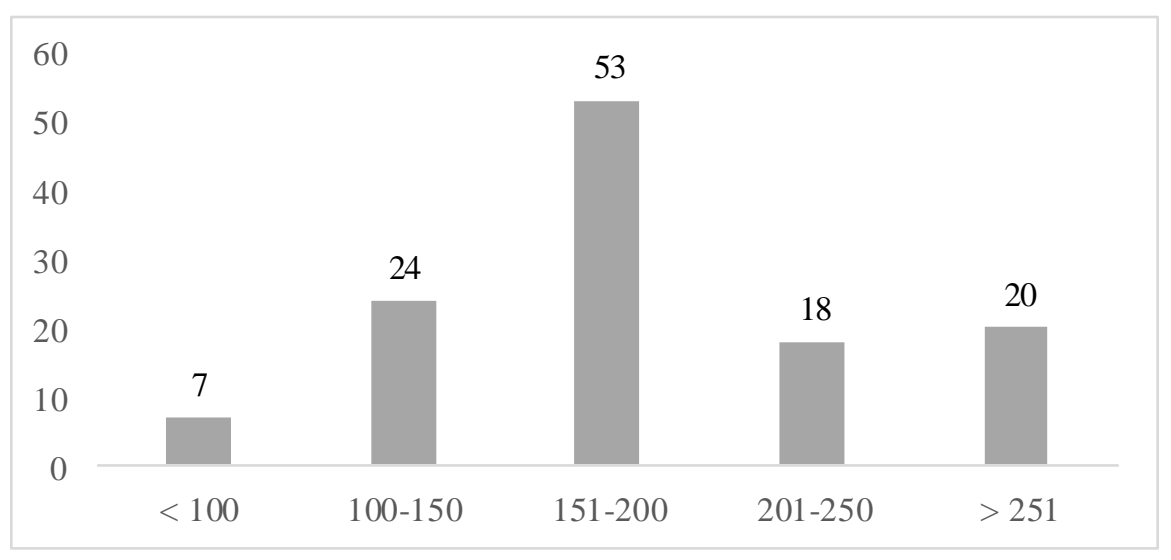

Quando avaliada a disciplina de farmacologia em relação a sua distribuição em aulas teóricas e/ou práticas, foi possível observar uma proporção maior $(68,0 \%)$ de instituições que oferecem apenas aulas teóricas (com uma média de carga horária de 213,19 h e desvio padrão de 120,13 h), em contraposição com o $32 \%$ das instituições que disponibilizam aulas teóricas e práticas (com uma média de carga horária de 190,85 h e desvio padrão de 66,05 h) em uma ou mais disciplinas de farmacologia (Figura 3).
Segundo Cardoso et al., (2011), esse dado pode estar relacionado com a dificuldade de desenvolvimento de aulas práticas, escassez de material didático para o desenvolvimento das práticas ou ainda, falta de interesse ou preparo dos docentes nessa área.

Todo ensino é baseado numa teoria, a qual visa o entendimento do estudante para com o contexto ao qual está prestes a se familiarizar. Porém, aliada à teoria estudada previamente, a prática é um dos momentos mais importantes para a formação 
profissional, pois é o momento de consolidação daquilo que foi teoricamente aprendido.

É nesse momento que o futuro profissional tem oportunidade de entrar em contato direto com a realidade profissional na qual será inserido, além de concretizar pressupostos teóricos adquiridos pela observação de determinadas práticas específicas e do diálogo com outros profissionais mais experientes ali presentes (técnicos de laboratório e professores, por exemplo).

Gráfico 3 - Tipo de aula ministrada nas disciplinas de Farmacologia dos Cursos Farmácia da região sudeste do Brasil.

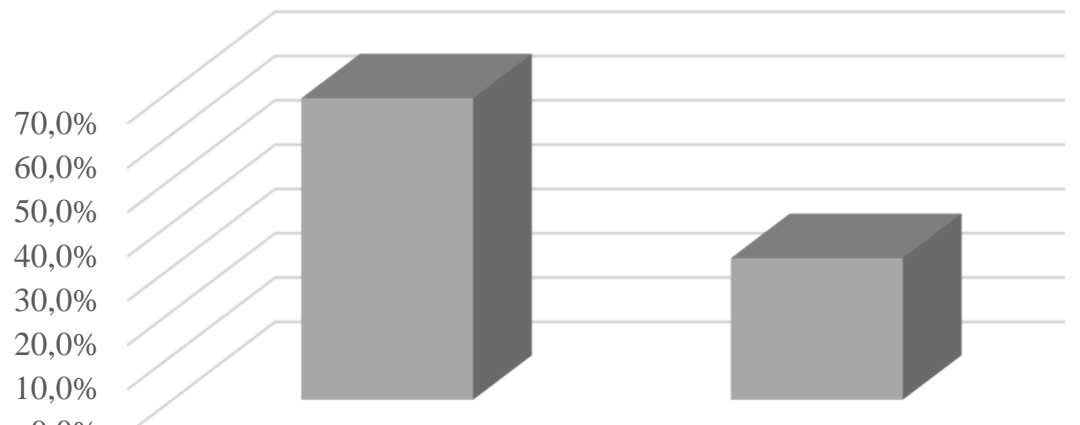

APENAS

Sendo assim, o desenvolvimento de TEÓRICA E

Esses dados mostram que a disciplina uma formação baseada no contexto real de é lecionada nos períodos intermediários do atuação possibilita a construção autônoma do conhecimento científico através da vivência de exemplos práticos para discussões acadêmicas. Na prática, o estudante tem a oportunidade de investigar, analisar e intervir na sua futura realidade profissional (BRASIL, 1996).

No que se refere à distribuição das disciplinas de Farmacologia segundo o período em que são ofertadas, foi possível observar que na sua maioria, estão inseridas entre o $4^{\circ}$ e $7^{\circ}$ períododa graduação. curso, e que não existe uma uniformidade na oferta dessa disciplina entre as instituições pesquisadas. É importante ressaltar que algumas instituições oferecem até 4 disciplinas relacionadas com a farmacologia, em contraste com outras que oferecem somente 1. As instituições que oferecem mais opções, consequentemente, se dispõem de um estudo diferenciado da farmacologia em cada disciplina ofertada, o que permite ao aluno o aprendizado da ciência vista por outros ângulos e aplicações. 
A farmacologia é uma das disciplinas de maior importância para farmacêuticos, que é o profissional que lidadiretamente com medicamentos, desde seu desenvolvimento farmacotécnico até o acompanhamento farmacoterapêutico dopaciente. Sendo assim, é de suma importânciaum planejamento para a oferta dessa disciplina nos cursos de graduação em Farmácia.

No momento do planejamento da oferta dessa disciplina, além da importância da carga-horária e da metodologia de ensino, é importante levar em consideração o amadurecimento do profissional em formação. Considerando o fato de que a farmacologia pode ser vista por vários ângulos, ou seja, desde sua base fisiológica até sua aplicação prática, faz-se necessário um aprendizado que também considera o amadurecimento do estudante enquanto profissional.

Visto isso, a base da disciplina de fato é mais aproveitada no início do curso, onde o estudante está lidando com conceitos básicos em todos os conteúdos que está aprendendo. Depois dessa parte, a disciplina já pode ser ensinada baseando-se no conteúdo já aprendido, com sua aplicação em casos clínicos, o que é capaz de começar a inserir o estudante na sua realidade profissional futura. Já no final do curso, onde o estudante já é praticamente um profissional, a inserção da farmacologia voltada para as disciplinas sociais, como Atenção e Semiologia Farmacêutica, permite que o conteúdo seja mais profundamente explorado e aproveitado pelo estudante, que já possui uma mente formada para sua atuação profissional.

Tabela 1 - Distribuição das disciplinas de Farmacologia segundo período em que são ofertadas, entre os Cursos de Farmácia da região sudeste do Brasil.

\begin{tabular}{|c|c|c|c|c|c|c|c|c|c|c|}
\hline \multirow[t]{2}{*}{ NOME DA DISCIPLINA } & \multicolumn{10}{|c|}{$\begin{array}{c}\text { NÚMERO DE INSTITUIÇÕES SEGUNDO } \\
\text { PERÍODO EM QUE A DISCIPLINA É } \\
\text { OFERTADA }\end{array}$} \\
\hline & $\mathbf{1}^{\mathbf{o}}$ & $2^{\circ}$ & $3^{\circ}$ & $4^{\circ}$ & $5^{\circ}$ & $6^{0}$ & $7^{\circ}$ & $\mathbf{8}^{\mathbf{0}}$ & $9^{\circ}$ & $10^{\circ}$ \\
\hline Farmacologia & & & 6 & 1 & 11 & 1 & & & & \\
\hline Farmacologia I & & 1 & 6 & 22 & 18 & 1 & & & & \\
\hline Farmacologia II & & & & 5 & 19 & 18 & 1 & & & \\
\hline Farmacologia III & & & & & 1 & 5 & 2 & 1 & & \\
\hline Farmacologia IV & & & & & & & 1 & & & \\
\hline Farmacologia Geral, Famacologia Básica, Farmacologia Clínica & & & 5 & 9 & 3 & 4 & 4 & 2 & & \\
\hline Farmacoterapêutica & & & & & & 1 & 1 & 1 & & \\
\hline Farmacoterapêutica I & & & & & 1 & & & & & \\
\hline Farmacodinâmica e Farmacocinética & & & & 2 & 2 & 1 & & & & \\
\hline Farmacoterapia & & & & 1 & & 3 & 3 & 1 & & 2 \\
\hline Farmacodinâmica & & & 1 & 3 & 1 & 5 & 1 & 1 & & \\
\hline Introdução à Farmacologia e História da Farmacologia & & & 1 & 1 & 1 & & & & & \\
\hline Farmacologia I e Farmacoepidemiologia & & & & 1 & & & & & & \\
\hline Farmacodinâmica I & & & & 2 & 8 & 2 & & & & \\
\hline Farmacodinâmica II & & & & & 2 & 8 & 2 & & & \\
\hline Farmacodinâmica III & & & & & & 1 & & 1 & & \\
\hline Farmacocinética e Farmacodinâmica I e II & & & & 2 & 4 & 2 & & & & \\
\hline Farmacocinética & & & & 1 & 3 & 1 & & & & \\
\hline
\end{tabular}




\begin{tabular}{|c|c|c|c|c|c|c|c|c|c|c|}
\hline Fisiopatologia e Farmacoterapia & & & 2 & & & & & 1 & & \\
\hline Fisiopatologia e farmacoterapia I & & & & & 10 & & & 1 & & \\
\hline Fisiopatologia e farmacoterapia II & & & & & & 10 & & & 1 & \\
\hline Fisiopatologia e farmacoterapia III & & & & & & & 8 & & & \\
\hline Fisiopatologia e farmacoterapia IV & & & & & & & 8 & & & \\
\hline Fisiopatologia e farmacoterapia V & & & & & & & 8 & & & \\
\hline Interações medicamentosas & & & & & 1 & & & 7 & & \\
\hline Tópicos em farmacologia clínica & & & & & & & & & & 1 \\
\hline Farmacologia farmacêutica I e II & & & & 1 & 1 & & & & & \\
\hline Farmacoepidemiologia & & & 1 & & & 1 & 2 & 3 & 1 & \\
\hline Farmacologia clínica e terapêutica & & & & & & & & 1 & & \\
\hline Introdução ao estudo de medicamentos & 1 & & & 1 & & & & & & \\
\hline Farmacologia Aplicada e Fundamentos de Farmacologia & & & & 2 & 2 & & 1 & & & \\
\hline Principios de farmacocinética e farmacodinâmica & & & & & 1 & & & & & \\
\hline Farmacologia do sistemas & & & & & 1 & & & & & \\
\hline Farmacoepidemiologia e Farmacovigilância & & & & & 1 & 2 & 2 & & & \\
\hline $\begin{array}{l}\text { Fisiopatologia e Farmacoterapia das Doen. Metab. Infec. e } \\
\text { Parasitárias }\end{array}$ & & & & & & & & & 1 & \\
\hline $\begin{array}{l}\text { Fisiopatologia e Farmacoterapia dos Distúrbios Hemat., Imun. e } \\
\text { Neop. }\end{array}$ & & & & & & & & & & 1 \\
\hline Bases Farmacoterapêuticas I, II e II & & & & & 1 & 1 & 1 & & & \\
\hline Farmacocinética e Farmacodinâmica Fundamental & & & & & 1 & & & & & \\
\hline Farmacologia Neuroendócrina & & & & & & 1 & & & & \\
\hline Farmacologia Cardiovascular e Quimioterápica & & & & & & & 1 & & & \\
\hline Farmacologia dos Sistemas I e II & & & & & & & 1 & 1 & & \\
\hline Farmacologia geral I e II & & & & & 1 & 1 & & & & \\
\hline Farmacovigilância & & & & & & & & 1 & & \\
\hline Famacocinética e Farmacodinâmica Aplicada & & & & & 1 & & & & & \\
\hline Farmacologia Integrada I e Farmacologia Integrada II & & & & & 1 & 2 & & & & \\
\hline Farmacologia sistêmica e Farmacologia Quantitativa & & 1 & & & 1 & & & & & \\
\hline Farmacologia Clínica e Terapêutica & & & & 1 & & & & & & \\
\hline $\begin{array}{l}\text { Estudo Integrado da Fisiopatologia e Farmacologia Terapêutica } \\
\text { I e II }\end{array}$ & & & & & 1 & 1 & & & & \\
\hline $\begin{array}{l}\text { Farmacologia nos Transtornos Menores e nos Transtornos } \\
\text { Maiores }\end{array}$ & & & & & & 1 & & & & \\
\hline Farmacocinética e Toxicocinética e Farmacogenética & & & & & 1 & 1 & & & & \\
\hline Total & 1 & 2 & 22 & 55 & 99 & 84 & 47 & 22 & 3 & 4 \\
\hline
\end{tabular}

\section{CONCLUSÃO}

A análise das informações obtidas com a pesquisa demonstraram a necessidade de uma reavaliação da grade curricular para a oferta de disciplinas relacionadas com a farmacologia e, consequente dos seus planos de ensino e ementa da disciplina, nos cursos de graduação em Farmácia do Sudeste do Brasil.

O perfil geral da oferta dessa disciplina que se faz de tanta importância para a formação do profissional farmacêutico mostrou defasagens quanto à diferença na carga-horária entre instituições, ausência de aulas práticas e distribuição da disciplina ao longo do curso, além da baixa oferta de disciplinas aplicadas à essa ciência, se restringindo, na sua maioria, à ciência básica somente.

Sendo assim, de acordo com o conjunto de resultados obtidos, sugere-se uma mudança na estrutura curricular dos cursos de graduação em Farmácia do Sudeste do Brasil, a fim de obter resultados mais satisfatórios no que diz respeito à preparação do profissional, 
levando em conta a farmacologia, que está prestes a ser inserido no mercado exercendo um papel vital na melhora da qualidade de vida da população que atende, sendo de forma indireta, na produção industrial e magistral, por exemplo, ou de forma direta, na orientação e indicação farmacêutica ao paciente.

\section{REFERÊNCIAS}

ADIB, L. Novos paradigmas sobre a atuação do profissional farmacêutico. Conferência de abertura do ano letivo de 2015 do curso de Farmácia da Universidade de Passo Fundo. Disponível em: < http://www.upf.br/site/inc/noticias/mostraNoti cia.php?codNoticia=24183 $>$. Acesso em 06 de janeiro de 2016.

BERMOND, M. D.; FERNANDES, Z. C.; COSTA, E. M. M.; CUNHA, N. S.; HONDA, A. M.Modelo referencial de ensino para uma formação farmacêutica com qualidade. Brasília: Conselho Federal de Farmácia, 114 p., 2008.

BRASIL. Lei n ${ }^{\circ}$ 9.394, de 20 de dezembro de 1996: Estabelece as diretrizes e bases da educação nacional. Brasília, DF, 1996.

Disponível em:

<http://portal.mec.gov.br/seesp/arquivos/pdf/l ei9394_ldbn1.pdf.>. Acesso em: 05 de janeiro de 2016.

BRASIL. Resolução CNE/CES n ${ }^{2}$, de 19 de fevereiro de 2002: Institui Diretrizes

Curriculares Nacionais do Curso de

Graduação em Farmácia. Brasília, DF, 2002.

Disponível em:

<http://portal.mec.gov.br/cne/arquivos/pdf/CE

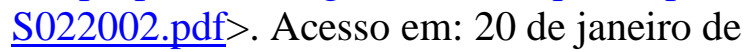
2016.

CARDOSO, L.; FREITAS, R. F.; PRAES, F.; JÚNIOR, W. P. Estudo da Disciplina de
Farmacologia em cursos de Farmácia no estado de Minas Gerais. Revista de Farmácia das Faculdades Santo Agostinho. v.1, n. 1, 2011.

DIAS, M. S. Farmacologia para

Farmacêuticos. Disponível em: <http://www.portaleducacao.com.br/farmacia/ artigos/20713/farmacologia-parafarmaceuticos $>$ Acesso em: 03 de janeiro de 2016.

SISTEMA e-MEC. Instituições de Educação Superior e Cursos Cadastrados. Disponível em: http://emec.mec.gov.br/. Acesso em: 15 de agosto de 2015.

FERNANDES, Z, C.; BERMOND, M, D.; FILHO, N. C . F. O.; CAMARGO, E. E. S.; CECY, C. Os Desafios da Educação Farmacêutica no Brasil.Brasília: Conselho Federal de Farmácia. 132p., 2008.

MOREIRA, F. A.; GUIMARÃES, F. S. Mecanismos de ação dos antipsicóticos: hipóteses dopaminérgicas. Medicina, Ribeirão Preto, v. 40, n. 1, p. 63-71,2007.

PESSOA, D. L. R.Perfil dos cursos de graduação em farmácia do nordeste brasileiro. Enciclopédia Biosfera, Centro Científico Conhecer. v. 6, n. 11, 2010.

RICIERI, M. C.; PREVIATTI, D.; CAMPESE, M.; CONSTANTINI, H. F.; MONTRUCCHIO, D. P.; KADES, A. S. O.; BUFFON, M. C. M.; FURMAN, I. M. O Farmacêutico no contexto da estratégia em saúde da família, que realidade é esta?Visão Acadêmica. v. 7, n. 2, 2006.

STOLF, A.; DREIFUSS, A.; VIEIRA, F. L.Farmacocinética. In: III Curso de Verão em Farmacologia. Universidade Federal do Paraná. Disponível em: $<$ http://insightltda.com.br/images/dinamica/pd f 7fe1310c8e894324299fcfd3b8f760ef.pdf $>$. Acesso em: 21 de janeiro de 2016. 